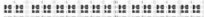

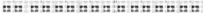

Heika Hoga

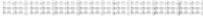

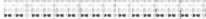

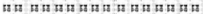

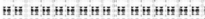

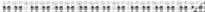

Architettura in Italia I valori e la bellezza

\author{
a cura di \\ Orazio Carpenzano \\ Dina Nencini \\ Manuela Raitano
}

I

หน

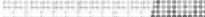

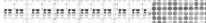

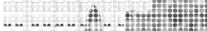

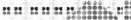
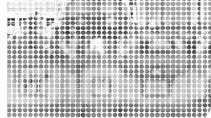

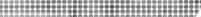

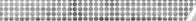

H:

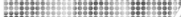

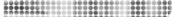

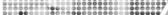

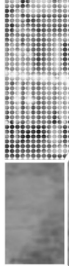

Quodlibet DiAP PRINT / TEORIE 14 
DiAP PRINT / TEORIE 14 



\section{Architettura in Italia I valori e la bellezza}

A cura di Orazio Carpenzano,

Dina Nencini, Manuela Raitano

Quodlibet 
DiAP Dipartimento di Architettura e

Progetto

Direttore Orazio Carpenzano

Sapienza Università di Roma

DIAP PRINT / TEORIE

Collana a cura del

Gruppo Comunicazione del DiAP

Coordinatore Cristina Imbroglini

COMITATO SCIENTIFICO

Carmen Andriani

Roberta Amirante

Jordi Bellmunt

Renato Bocchi

Giovanni Corbellini

Giovanni Durbiano

Carlo Gasparrini

Sara Marini

Luca Molinari

Alessandra Muntoni

Franco Purini

Joseph Rykwert

Andrea Sciascia

Zeila Tesoriere

Ilaria Valente

Herman van Bergeijk

Franco Zagari

Ogni volume della collana è sottoposto alla revisione di referees esterni al Dipartimento di Architettura e Progetto scelti tra i componenti del Comitato Scientifico.
(C) 2018

Quodlibet srl via Giuseppe e Bartolomeo Mozzi, 23 Macerata

www.quodlibet.it

PRIMA EDIZIONE

aprile 2018

ISBN

978-88-229-0187-3

IN COPERTINA

Elaborazione grafica di Mariangela Ludovica Santarsiero 
Indice

9 Nota dei curatori. Le ragioni di un incontro

I3 Preludio

Orazio Carpenzano

25 Valori e bellezza. Contemporanee fertili inquietudini e necessarie azioni

Dina Nencini

33 Via il piombo dalle ali! Manuela Raitano

Parte prima. Di bellezze e di valori

45 Il "politeismo dei valori” dell'architettura italiana Marco Biraghi

53 Fuori dalla crisi: rospi, bellezze e il sapere dell'architetto Roberta Amirante

63 La bellezza è un fraintendimento Carmen Andriani

77 Salvare la bellezza. L'architettura italiana al bivio Luca Molinari

87 La bellezza come disvelamento della natura delle cose Carlo Moccia 
INDICE

95 L'architettura come arte positiva

Franco Purini

I07 L'architettura è solo per una stirpe felice?

Anna Irene Del Monaco

I 2 I Bellezza e commisurazione

Mariangela Ludovica Santarsiero

I 29 L'esperienza complessa dei corpi tra mente e ambiente. Architettura abito del corpo

Giovanni Rocco Cellini

I37 Ordine e disordine, armonie e dissonanze

Alessandra Capanna

I47 Bellezza o dello sguardo critico

Antonino Saggio

I 55 Legami ambigui e necessari tra bellezza e valori

Luca Porqueddu

I65 La bellezza come diritto e come espressione di dignità civile Piero Ostilio Rossi

I77 Il paesaggio tra valori e bellezza

Alessandra Capuano

Parte seconda. Architetture in Italia

I 89 Dieci note per una bellezza possibile

Michele Beccu

20I L'indicibilità della bellezza e l'appropriatezza della costruzione Bruno Messina

2 I I Architettura e tecnica di guerriglia

Cherubino Gambardella 
2 I 7 Le idee e il progetto

Vittorio Pizzigoni

225 Lo spazio pubblico come valore. Figure della bellezza negli spazi della mobilità

Filippo Lambertucci

235 La ricerca dell'aura perduta

Aldo Aymonino

Parte terza. Letture

245 Archeologia del bello

Anna Giovannelli

255 Tra valore d'uso e funzione

Lelio Di Loreto

265 Valori mutevoli nelle case di vacanza di Gio Ponti

Pisana Posocco

277 La bellezza ha uno spirito interiore Simona Ottieri

283 Valori e bellezze negli interventi di piccola scala Gianpaola Spirito

29 I Il diritto al brutto di Lina Bo Bardi e la bellezza dell'architettura popolare

Alessandra Criconia

303 Effetti collaterali

Edoardo Albinati 



\title{
Ordine e disordine, armonie e dissonanze
}

\author{
Alessandra Capanna
}

\section{Una premessa teorica}

Quasi tutti sanno distinguere, in modo intuitivo, cosa sia un evento ordinato o casuale; il modo più semplice è quello di definire, secondo un approccio istintivamente geometrico, la posizione fisica degli oggetti o delle loro parti; poiché la geometria, come coacervo di relazioni di posizione, quantità e qualità, riguarda l'agire umano, che si svolge principalmente secondo schemi organizzati, orientati nello spazio e nel tempo.

Questa riflessione prende le mosse da un'affermazione di Hilberseimer a proposito della realtà metropolitana: "la sfumatura si cancella, regna la misura, che costringe il caos a diventare forma, forma logica, univoca, matematica", e affronta il tema dell'armonia e della dissonanza nella genesi compositiva, per ragionare sulle leggi che regolano "ordini" di tipo complesso. Un qualche rudimento d'ordine infatti sembra essere sempre presente, sebbene sia facile giudicare caotici, per esempio, la crescita delle città, la vita moderna, il traffico, le grezze agglomerazioni di forze non coordinate. Intervengono, nell'osservazione di tali eventi, considerazioni di carattere percettivo e soggettivo che conducono la riflessione nel campo della psicologia della Gestalt ${ }^{1}$, che però ci informa che il caos non può essere visto finché si osserva la realtà dal basso, come costituita da frammenti i cui rapporti reciproci spesso si fermano alla relazione con il frammento più vicino. $\mathrm{E}$ certamente, da un simile punto di vista, anche l'ordine rimarrà nascosto. Occorre perciò prendere le distanze e rilevare le strut-

\footnotetext{
${ }^{\text {I }}$ M. Wertheimer, Gestalt Theory, "Social Research”, I I, I, febbraio I944.
} 
ture da lontano, operazione che consente di cogliere schemi e ritmi, misure e rapporti reciproci che, secondo Arnheim², determinano inequivocabilmente relazioni d'ordine.

Impostare il ragionamento conferendo in modo preventivo connotazioni positive all'attributo di ordine vuol dire anche prendere una posizione decisa sulla contemporaneità. Valutare quando, e se, schemi compositivi regolari e regolati siano stati sostituiti dai loro opposti, per quale motivo, e se questo abbia comportato un cambiamento nel nostro giudizio estetico.

\section{Alcune considerazioni (multi) disciplinari}

Bisogna innanzitutto ricordare che alcuni teorici dell'informazione e della percezione estetica affermano che l'arte si trova nel punto di passaggio tra ordine e disordine, intendendo con arte un fenomeno globale, facente parte dei processi di strutturazione - pertanto compositivi - che comprendono ogni tipo di attività poetica.

Arnheim sosteneva invece, come di seguito è riportato, che la negazione di queste relazioni d'ordine, attuata per esempio da Robert Venturi come ribellione nei confronti dell'ascetica sobrietà del Bauhaus e dell'International Style, rappresentasse in sé un oltraggio:

La contraddizione è un difetto. [...]

Ciò che Venturi ha in mente, costituisce un affronto all'ordine. [...] Quando si verifica in una asserzione, consiste nell'attribuire a un oggetto $\mathrm{o}$ a un fatto un particolare predicato, che però in pari tempo viene negato. Si tratta di una violazione della regola del terzo escluso, per la quale ogni predicato deve essere o attribuito o negato a qualunque cosa. Si è in errore allorché si asserisce che qualcosa esiste e non esiste nel medesimo tempo. Né è possibile che una cosa possieda nello stesso luogo e tempo due proprietà che reciprocamente si escludono. Un oggetto può certamente essere in parte rosso e in parte blu, o sembrare blu al mattino e rosso alla sera, oppure essere ritenuto blu da una persona e rosso da un'altra. Ma le stesse parti non possono risultare sia rosse che

${ }^{2}$ Cfr. R. Arnheim, La dinamica della forma architettonica, Feltrinelli, Milano I98 I, in part. cap. 6 . 
blu nelle medesime condizioni, per quanto l'oggetto possa comporsi di una qualche mescolanza dei due colori. Esso può inoltre assolvere svariate funzioni, purché queste siano compatibili tra loro. Può anche essere ambiguo, nel senso che vi si alternano entrambe le versioni. Tutti questi sono generi possibili e accettabili di ordine. Ma se di una cosa dite che è questo e anche quello, e l'uno e l'altro si escludono a vicenda, ciò che si crea è solo confusione e l'ordine diventa disordine ${ }^{3}$.

L'intrigante testo di Arnheim fornisce spunti di riflessione sull'evoluzione del pensiero scientifico; la debolezza delle sue argomentazioni sulla complessità e sul disordine deriva da un uso stretto della Logica, disciplina che invece, approssimativamente settant'anni prima che venisse pubblicato La dinamica della forma architettonica, aveva già decretato il superamento del principio del terzo escluso, citato nel brano sopra riportato.

La logica aristotelica si basava infatti su tre pilastri: le leggi di identità, di contraddizione e del terzo escluso. Quest'ultima legge non è valida però nelle logiche a tre valori, nate all'inizio del nostro secolo, per le quali una data proposizione può essere vera, falsa, indeterminata ${ }^{4}$, oppure vera, falsa, possibile; come ovviamente non è valida neppure per quelle logiche che ammettono $n$ valori di verità, che sono alla base di molti studi sull'intelligenza artificiale e che analizzano varie alternative possibili all'interno di sistemi complessis.

${ }^{3}$ Ivi, p. I 85 .

${ }^{4}$ Per essere più precisi il superamento del principio di esclusione ammette che una proposizione sia vera, falsa, né vera né falsa. Nella formulazione del filosofo matematico A. Heiting si incontra la dizione: vero-falso-indeterminato, mentre in quella di J. Lukasiewicz e di A. Tarski: vero-falso-possibile.

5 Alfred Tarski, filosofo della matematica dell'inizio del '900, giunse alla definizione della nozione di più verità studiando le possibilità di esprimere il concetto di verità aritmetica all'interno della disciplina matematica che va sotto il nome di Aritmetica Tipografica. Senza entrare nel campo specifico che esula dai limiti della presente analisi, la formula di Tarski, che verte sugli enunciati contemporaneamente veri e falsi, in sintesi, riproduce il paradosso di Epimenide, o paradosso del mentitore. Epimenide era un cretese che pronunciò la nota frase: "Tutti i cretesi sono mentitori", che in una versione più incisiva può essere tradotta in: "Io sto mentendo", oppure nel paradosso logico-matematico; "Questo enunciato è falso". L'elaborazione figurativa di questa formulazione è perfettamente riprodotta in due disegni di Escher: Cascata e Salita e discesa, ove è contemporaneamente vero e falso che stiamo osservando un percorso che ascende. 
La logica a tre valori è più interessante al fine di individuare nuovi ordini possibili. Essa nasce all'interno della scuola “intuizionista" fondata all'inizio del '90o da Luitzen Egbertus Jan Brouwer, un matematico olandese noto per le sue ricerche sull'applicazione della matematica ai diversi aspetti della realtà.

La logica dell'intuizionismo prende le mosse dall'idea kantiana circa le intuizioni umane fondamentali dello spazio e del tempo, che determinano nella nostra mente la conoscenza dei singoli oggetti grazie alla capacità innata di percepire qualcosa, senza dover cogliere ogni aspetto della sua struttura.

Kant, però, faceva distinzione tra una percezione di tipo spaziale e una di tipo temporale: associava la geometria all'intuizione spaziale, mentre associava l'aritmetica e il contare sequenziale alla percezione temporale. Ma le idee di Kant facevano riferimento alla geometria euclidea, considerata latrice, ai suoi tempi, di verità assoluta, mentre Brouwer aveva approfondito gli studi sulla geometria topologica che si può dire riesca ad unificare i due ambiti (spaziale e temporale) come conseguenza di un processo di deformazione. Se quindi, in astratto, la sua logica a tre valori ammetteva che un concetto possa essere vero, falso, indeterminato, lo stesso ragionamento può tradursi nell'ambito topologico in vero, falso, deformato. Per fare un esempio, il principio del terzo escluso avrebbe definito una forma geometrica $A$, o non- $A$, e cioè: o si tratta di un cerchio oppure di un'ellisse. Ma l'ellisse $\grave{e}$ il cerchio deformato topologicamente, per cui può senz'altro ammettersi, nella nuova geometria topologica, la possibilità che una cosa sia questo e quello contemporaneamente, A e non-A. Soprattutto, ragionando sulla complessità di oggetti posti nello spazio che abbiamo imparato a conoscere grazie all'apporto teorico della relatività di Einstein, possiamo ammettere una quarta dimensione che è lo spazio-tempo ${ }^{6}$. Le distinzioni einsteiniane sui vari significati dello spazio in fisica riguardano il concetto di spazio come luogo identificabile; il concetto di spazio assoluto;

${ }^{6}$ Le implicazioni degli studi fisici sulla relatività con quelli relativi alle geometrie non-euclidee sono esaminate ed esposte con chiarezza nel testo, fondamentale per l'approccio matematico alla conoscenza, di M. Kline, La matematica nella cultura occidentale, Feltrinelli, Milano I976, in part. cap. 27, "La teoria della relatività". 
il concetto di spazio relativo quadridimensionale. Trasponendo questi principi in architettura, da una parte l'assoluta relatività del concetto di luogo va sostituendo gradualmente quello di spazio, definendo, tra l'altro, una sempre maggiore smaterializzazione della nozione di stile a favore di un altro valore, quello della a-scalarità, di grande potenziale figurativo; dall'altra, l'unità spazio-plastica delle masse corporee interpreta la quarta dimensione dello spazio-tempo come una delle fasi della percezione della realtà dell'architettura.

Se quindi non è corretto giudicare disordinati i sistemi la cui complessità deriva dal fatto di rispondere a leggi derivanti da altri tipi di ordine, è però senz'altro vero che la vista e il movimento all'interno dello spazio fisico si sono assuefatti a svolgersi in luoghi che rispondono alle leggi della geometria euclidea, che, se così si può dire, definisce un ordine di tipo semplice, fatto di equilibri e simmetrie che risultano positivi per ragioni derivanti dal rapportarsi in modo diretto alla sfera psicologica e fisiologica. Più forte infatti sembra essere stato, per lungo tempo, il bisogno di stabilire leggi che conducano a immagini ordinate, nella convinzione che queste sole determinino la soddisfazione dello spirito.

Attraverso il progetto come costruzione, da parte dell'uomo, del suo proprio universo, sono invece, in definitiva, ammissibili tutti i tipi di ordine perseguibili tramite l'astrazione geometrica, che è strumento proprio della costruzione del disegno architettonico.

Quello, insomma, che preme sottolineare è la possibilità di individuare una forma logica che sia alla base della struttura geometrica della composizione nelle sue trasformazioni, nelle discontinuità, nelle interruzioni, nei passaggi, nei confini di una regola, nella forza di propagazione e nella capacità di coesistenza e di sovrapposizione con le figure di riferimento.

Anche la composizione della musica è sottoposta alla medesima revisione; l'effetto rivoluzionario del rifiuto dei rapporti gerarchici nella costruzione della struttura dodecafonica si rivela nell'individuazione di un sistema di regole ancor più rigido che nella sostanza affronta il problema compositivo in un'ottica di vero e proprio relativismo. 
1. Logica sfumata (elaborazione digitale di A. Capanna).

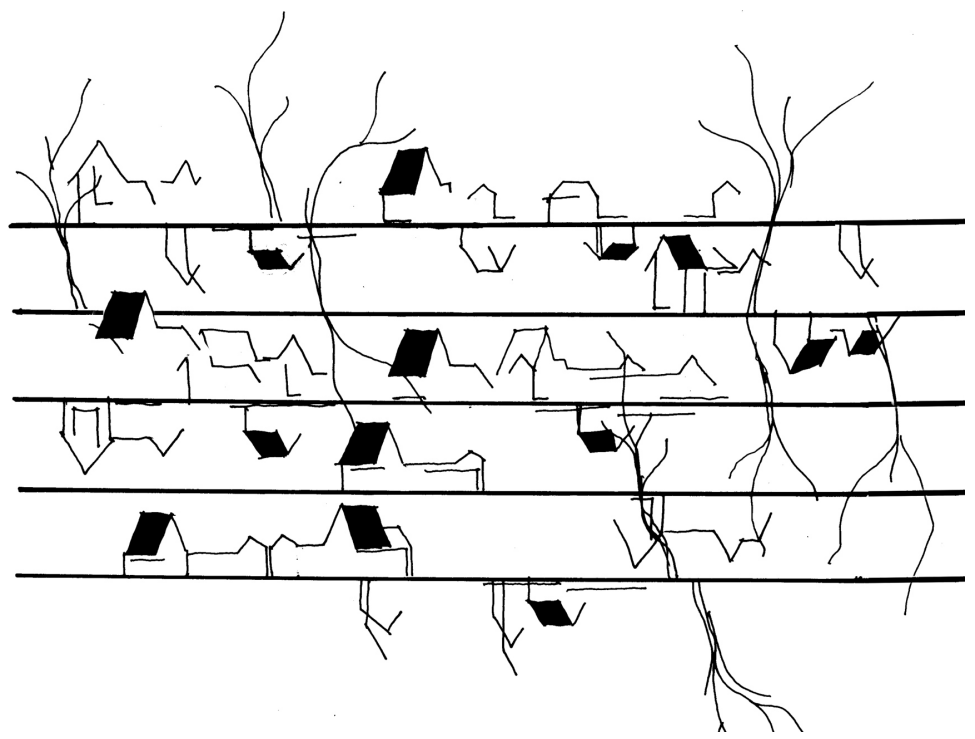

Per volontà e non per caso

Le espressioni "consonanza" e "dissonanza" che indicano un'antitesi sono errate [superamento del principio del terzo escluso]: dipende solo dalla crescente capacità dell'orecchio di familiarizzarsi anche con gli armonici più lontani, allargando in tal modo il concetto di suono atto a produrre un effetto d'arte in modo che vi trovi posto tutto il fenomeno naturale nel suo complesso?.

Non è difficile individuare nell'architettura del primo '900 opere che rispecchiano gli stessi principi di atonalità della musica dodecafonica; in molti hanno sottolineato la presenza di tale criterio compositivo nel Raumplan di Loos ${ }^{8}$, che si fonda infatti, tra l'altro, sul superamento della concezione classica dello spazio gerarchicamente ordinato.

Ma ancora più interessante è l'atonalità nell'organizzazione degli spazi della costruzione tradizionale giapponese, i cui

7 A. Schönberg, Manuale di armonia, il Saggiatore, Milano I963, p. 24.

${ }^{8}$ Cfr., tra gli altri contributi sull'argomento, l'esaustivo articolo di A. Melchiorri, Schönberg tra Loos e Kandinsky, "Casabella", numero monografico su Architettura e Musica, 473, I98I. 
elementi costitutivi sono del tutto indifferenziati: i tatami generano non più una disposizione gerarchica globale, ma una molteplicità di gerarchie locali e frammentarie ed una generale estetica dell'asimmetria, voluta per giustapposizione di ordini diversi, fortemente influenzati dalla filosofia Zen, secondo la quale la bellezza risiede nella sua imperfezione.

Il "grande vano" di tutta l'arte giapponese si esprime nell'architettura della continuità dello spazio, fatta di soffitti e di struttura ineguali - del tutto analoghi, ad una osservazione comparata e a-storica, al loosiano Raumplan - con divisioni mobili che rendono possibili diverse misure e diverse forme dei vuoti, con aperture verso l'esterno di disegno e grandezze variabili, senza alcuna ricerca della regolarità e dell'equilibrio speculare della costruzione geometrica delle pareti.

Esempio simbolo di questa estetica è il Padiglione del Tè, o stanza dell'asimmetria, nel giardino della villa imperiale di Katsura, alle porte di Kyoto, le cui trame disuguali sono un anelito alla perfezione della libertà della vita interiore, sintetizzata in un'architettura che comprende scorci del paesaggio ed improvvise forme libere, come i pilastri a forma di tronco d'albero innestati nella modulazione regolare di suoli e i recinti derivanti dall'iterazione del modulo-tatami.

Argomento della progettazione sono, in definitiva, i comportamenti e i pensieri umani, eventi fatti di spazio e di tempo inseparabili nella concretezza dell'esperienza reale, sia nei luoghi simbolici della contemplazione che nell'edilizia abitativa comune, ove la percezione della stanza comincia dalla collina che si vede all'orizzonte ed è fatta di ritmi di luce e ombra nel fluire temporale delle immagini.

\section{Un epilogo provvisorio}

In linea di principio, perciò, non è errato individuare le regole delle diverse geometrie per definire l'ordine delle composizioni, ma è anche facile notare che tale operazione è descrittiva di singoli avvenimenti e relativa al punto di vista e al giudizio 


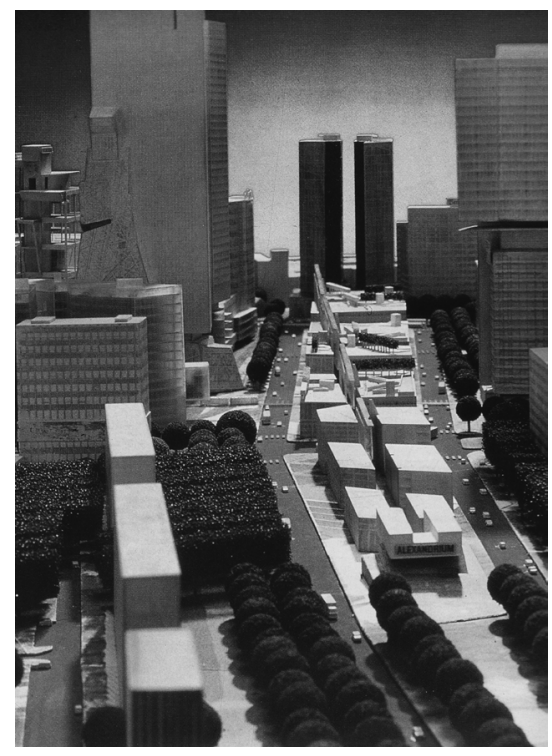

2. Daniel Libeskind, progetto per l'Alexanderplatz, Berlino, 1990.

personale, collegato alle leggi generative, aspetti che sono mutevoli, non sono assoluti e di conseguenza sono insufficienti a definire in modo univoco la differenza tra ordine e caos.

Alcune interessanti conseguenze risultano, allora, dall'osservazione della definizione algoritmica di numero ordinato o casuale 9 : innanzitutto la quantità di informazione necessaria per esprimere un numero sarà direttamente proporzionale al suo aumento di complessità. L'ordine di grado più semplice sarà rappresentato da una successione identica di elementi oI, per cui sarà sufficiente il massimo della sintesi espressiva: ripeti $n$ volte. Nella composizione degli spazi dell'architettura la teoria dell'informazione consente livelli di comunicazione degli oggetti architettonici sempre meno sintetici, a partire da quelli caratterizzati da semplici moduli ripetuti, fino a giungere al caso dell'impossibilità di fornire una forma (= in-formare) verbale di ciò che è composto secondo principi di deformazione tanto complessa da rendere necessaria l'esperienza percettiva completa per avere conoscenza dell'oggetto. In altre parole, il modo più semplice di informare su un'architettura caratterizzata, per esempio, da una forte deformazione topologica o addirittura da caotiche agglomerazioni di elementi, risulta essere la sua rappresentazione fisica per intero.

Questo metodo consente la definizione, libera da giudizi qualitativi e perciò vicina all'obiettività, del livello di complessità dell'oggetto, e quanto più lo schema strutturale risulterà nascosto,

${ }_{9}$ Se espressi nella loro forma binaria saranno evidenti le caratteristiche regolari di un numero formato da una successione orororor e meno evidenti quelle di un numero composto da da una successione del tutto casuale come, per esempio, OI IOI IOOOIO I I I I, ottenuta lanciando in aria una moneta e scrivendo I ogni volta che viene testa e o se risulta croce. 
tanto più complessa sarà la sua comunicazione. Il limite di incomprimibilità dell'informazione del sistema casuale, inoltre, sembrerebbe non comportare come conseguenza la totale assenza di un principio formale, perché quello casuale è pur sempre un criterio, soprattutto se ci si riferisce al campo della produzione artistica.

La composizione atonale rappresenta quindi, per l'Occidente moderno, l'intuizione, l'ammissione e la codificazione di un nuovo modello di ordine adeguato ai risultati del progresso scientifico, e la premessa necessaria per il successivo passaggio alla composizione aleatoria. Questi modelli teorici in architettura ancora devono trovare una declinazione appropriata e di piena consapevolezza. Essi però possono comunque ricadere in quella che Zevi chiama "seconda invariante dell'architettura", ovvero l'asimmetria e la dissonanza, che sintetizza il passaggio dal codice classico a quello moderno, in virtù dell'applicazione volontaria di un metodo progettuale che da un lato è il risultato di catene illogiche prodotte da libere associazioni mentali e dall'altro della logica della deformazione, come caso particolare della variazione del tema, dell'ordine topologico, della decostruzione.

Il lavoro di Daniel Libeskind degli anni '9o, in tal senso, è paradigmatico; egli stesso riconosce quale spunto iniziale un assioma di Paul Valéry: "l'umanità è perennemente minacciata da due pericoli: l'ordine e il disordine". Termini opposti tra i quali si forma una volontà espressiva tesa alla rappresentazione della trasformazione dello spazio urbano, che solo apparentemente rifiuta la lettura storica dei luoghi per una più forte interpretazione dell'architettura come atopica spettacolarizzazione dello spazio-tempo.

La città è la più grande creazione spirituale dell'umanità: un lavoro collettivo che valorizza l'espressione di una cultura, di una società e dell'individuo nel tempo e nello spazio. La struttura è intrinsecamente misteriosa. [...] nel progetto per Berlino-Alexanderplatz ho suggerito la matrice aperta e sempre diversa che rafforza processi di trasformazione e vede il dinamismo del cambiamento in una architettura diversa e pluralistica ${ }^{10}$.

${ }^{10}$ La citazione è tratta dalla relazione di D. Libeskind al concorso per BerlinoAlexanderplatz, riportata nel numero monografico di "L'Architettura", 465-466, I994. 
A Berlino Libeskind propone una città non più governata da regole urbanistiche, ma formata da una sequenza di immagini mutevoli e discontinue. Nel disordine di questo paesaggio è evidente che l'oggetto architettonico debba trovare al suo interno un principio narrativo. Da una parte adottando il collage come regola compositiva, dall'altra la memoria del futuro come fine.

Se quindi la città del Moderno era, "nonostante tutto, espressione di una lotta disperata per il dominio sul divenire" (Dal Co), ora ci troviamo in uno spazio urbano nel quale il flusso evolutivo ha subito un'interruzione netta e duratura, iniziata teoricamente nella prima metà del secolo con l'inserimento ideale del Plan Voisin lecorbusieriano all'interno del tessuto parigino.

E dunque, una più generale definizione di cosa sia una composizione ordinata si potrà in conclusione desumere dalle seguenti riflessioni di Schönberg:

È nostro dovere meditare continuamente sulle cause di ogni risultato artistico, senza mai stancarci di ricominciare da principio, sempre osservando e sempre cercando un nostro ordine, considerando come elementi dati solo i fenomeni, i fatti artistici, i quali possono essere ritenuti stabili a maggior diritto di ogni famoso discorso intorno all'arte. Poiché li conosciamo con certezza, avremo diritto di chiamare "scienza" quel che sappiamo di loro. Osservare una serie di opere, classificarle secondo alcune caratteristiche comuni, dedurne le leggi. Questo è giusto, non fosse altro per il fatto che non esistono altre possibilità. Tali leggi sono vere per le opere osservate fino a quel momento; possono non più esserlo per le opere future ${ }^{\mathrm{I}}$.

${ }^{11}$ A. Schönberg, Stile e idea, Rusconi e Paolazzi, Milano I960. 